\title{
On the size of $\left(K_{t}, \mathcal{T}_{k}\right)$-co-critical graphs
}

\author{
Zi-Xia Song* Jingmei Zhang \\ Department of Mathematics \\ University of Central Florida \\ Orlando, FL 32816, U.S.A. \\ Zixia.Song@ucf.edu jmzhang@Knights.ucf.edu
}

Submitted: Jul 5, 2019; Accepted: Nov 24, 2020; Published: Jan 15, 2021

(C) The authors. Released under the CC BY-ND license (International 4.0).

\begin{abstract}
Given an integer $r \geqslant 1$ and graphs $G, H_{1}, \ldots, H_{r}$, we write $G \rightarrow\left(H_{1}, \ldots, H_{r}\right)$ if every $r$-coloring of the edges of $G$ contains a monochromatic copy of $H_{i}$ in color $i$ for some $i \in\{1, \ldots, r\}$. A non-complete graph $G$ is $\left(H_{1}, \ldots, H_{r}\right)$-co-critical if $G \nrightarrow\left(H_{1}, \ldots, H_{r}\right)$, but $G+e \rightarrow\left(H_{1}, \ldots, H_{r}\right)$ for every edge $e$ in $\bar{G}$. In this paper, motivated by Hanson and Toft's conjecture [Edge-colored saturated graphs, J Graph Theory 11(1987), 191-196], we study the minimum number of edges over all $\left(K_{t}, \mathcal{T}_{k}\right)$-co-critical graphs on $n$ vertices, where $\mathcal{T}_{k}$ denotes the family of all trees on $k$ vertices and $G \rightarrow\left(K_{t}, \mathcal{T}_{k}\right)$ if for every 2-coloring $\tau: E(G) \rightarrow$ \{red, blue\}, $G$ has a red $K_{t}$ or a blue tree in $\mathcal{T}_{k}$. Following Day [Saturated graphs of prescribed minimum degree, Combin. Probab. Comput. 26 (2017), 201-207], we apply graph bootstrap percolation on a not necessarily $K_{t}$-saturated graph to prove that for all $t \geqslant 4$ and $k \geqslant \max \{6, t\}$, there exists a constant $c(t, k)$ such that, for all $n \geqslant(t-1)(k-1)+1$, if $G$ is a $\left(K_{t}, \mathcal{T}_{k}\right)$-co-critical graph on $n$ vertices, then

$$
e(G) \geqslant\left(\frac{4 t-9}{2}+\frac{1}{2}\left\lceil\frac{k}{2}\right\rceil\right) n-c(t, k) .
$$

Furthermore, this linear bound is asymptotically best possible when $t \in\{4,5\}$ and $k \geqslant 6$. The method we develop in this paper may shed some light on attacking Hanson and Toft's conjecture.
\end{abstract}

Mathematics Subject Classifications: 05C55, 05C35

\section{Introduction}

All graphs considered in this paper are finite, and without loops or multiple edges. For a graph $G$, we will use $V(G)$ to denote the vertex set, $E(G)$ the edge set, $|G|$ the number

*Supported by the National Science Foundation under Grant No. DMS-1854903. 
of vertices, $e(G)$ the number of edges, $N_{G}(x)$ the neighborhood of vertex $x$ in $G, \delta(G)$ the minimum degree, $\Delta(G)$ the maximum degree, and $\bar{G}$ the complement of $G$. If $A, B \subseteq$ $V(G)$ are disjoint, we say that $A$ is complete to $B$ if every vertex in $A$ is adjacent to every vertex in $B$; and $A$ is anti-complete to $B$ if no vertex in $A$ is adjacent to a vertex in $B$. The subgraph of $G$ induced by $A$, denoted $G[A]$, is the graph with vertex set $A$ and edge set $\{x y \in E(G): x, y \in A\}$. We denote by $B \backslash A$ the set $B-A, e_{G}(A, B)$ the number of edges between $A$ and $B$ in $G, e_{G}(A)$ the number of edges of $G[A]$, and $G \backslash A$ the subgraph of $G$ induced on $V(G) \backslash A$, respectively. If $A=\{a\}$, we simply write $B \backslash a$, $e_{G}(a, B)$, and $G \backslash a$, respectively. For any edge $e$ in $\bar{G}$, we use $G+e$ to denote the graph obtained from $G$ by adding the new edge $e$. The join $G+H$ (resp. union $G \cup H$ ) of two vertex disjoint graphs $G$ and $H$ is the graph having vertex set $V(G) \cup V(H)$ and edge set $E(G) \cup E(H) \cup\{x y: x \in V(G), y \in V(H)\}$ (resp. $E(G) \cup E(H)$ ). Given two isomorphic graphs $G$ and $H$, we may (with a slight but common abuse of notation) write $G=H$. For an integer $t \geqslant 1$ and a graph $H$, we define $t H$ to be the union of $t$ disjoint copies of $H$. We use $K_{n}$ and $T_{n}$ to denote the complete graph and a tree on $n$ vertices, respectively. For any positive integer $r$, we write $[r]$ for the set $\{1,2, \ldots, r\}$. We use the convention " $A:=$ " to mean that $A$ is defined to be the right-hand side of the relation.

Given an integer $r \geqslant 1$ and graphs $G, H_{1}, \ldots, H_{r}$, we write $G \rightarrow\left(H_{1}, \ldots, H_{r}\right)$ if every $r$-coloring of $E(G)$ contains a monochromatic $H_{i}$ in color $i$ for some $i \in[r]$. The classical Ramsey number $R\left(H_{1}, \ldots, H_{r}\right)$ is the minimum positive integer $n$ such that $K_{n} \rightarrow\left(H_{1}, \ldots, H_{r}\right)$. Following Nešetřil [13], and Galluccio, Simonovits and Simonyi [10],

Definition 1. A non-complete graph $G$ is $\left(H_{1}, \ldots, H_{r}\right)$-co-critical if $G \nrightarrow\left(H_{1}, \ldots, H_{r}\right)$, but $G+e \rightarrow\left(H_{1}, \ldots, H_{r}\right)$ for every edge $e$ in $\bar{G}$.

It is simple to check that $K_{6}^{-}$is $\left(K_{3}, K_{3}\right)$-co-critical, where $K_{6}^{-}$denotes the graph obtained from $K_{6}$ by deleting exactly one edge. It is worth noting that every $\left(H_{1}, \ldots, H_{r}\right)$ co-critical graph has at least $R\left(H_{1}, \ldots, H_{r}\right)$ vertices.

Remark. Following Galluccio, Simonovits and Simonyi [10], we exclude the complete graphs in the definition of $\left(H_{1}, \ldots, H_{r}\right)$-co-critical graphs, else every complete graph on fewer than $R\left(H_{1}, \ldots, H_{r}\right)$ vertices is $\left(H_{1}, \ldots, H_{r}\right)$-co-critical.

The notation of co-critical graphs was initiated by Nešetřil [13] in 1986 when he asked the following question regarding $\left(K_{3}, K_{3}\right)$-co-critical graphs:

Are there infinitely many minimal co-critical graphs, i.e., co-critical graphs which lose this property when any vertex is deleted? Is $K_{6}^{-}$the only one?

This was answered in the positive by Galluccio, Simonovits and Simonyi [10]. They constructed infinitely many minimal $\left(K_{3}, K_{3}\right)$-co-critical graphs without $K_{5}$ as a subgraph. Szabó [15] then constructed infinitely many nearly regular $\left(K_{3}, K_{3}\right)$-co-critical graphs with 
low maximum degree. It remains unknown whether there are infinitely many strongly minimal co-critical graphs, where an $\left(H_{1}, \ldots, H_{r}\right)$-co-critical graph is strongly minimal cocritical if it contains no proper subgraph which is also $\left(H_{1}, \ldots, H_{r}\right)$-co-critical. This is one of the most intriguing open problems proposed by Galluccio, Simonovits and Simonyi in [10]. One interesting observation made in [10] is that if $G$ is $\left(H_{1}, \ldots, H_{r}\right)$-co-critical, then $\chi(G) \geqslant R\left(H_{1}, \ldots, H_{r}\right)-1$. They also made some observations on the minimum degree of $\left(K_{3}, K_{3}\right)$-co-critical graphs and maximum number of possible edges of $\left(H_{1}, \ldots, H_{r}\right)$-cocritical graphs.

We want to point out here that Hanson and Toft [12] in 1987 also studied the minimum and maximum number of edges over all $\left(H_{1}, \ldots, H_{r}\right)$-co-critical graphs on $n$ vertices when $H_{1}, \ldots, H_{r}$ are complete graphs, under the name of strongly $\left(\left|H_{1}\right|, \ldots,\left|H_{r}\right|\right)$-saturated graphs. Recently, this topic has been studied under the name of $\mathcal{R}_{\min }\left(H_{1}, \ldots, H_{r}\right)$ saturated graphs $[5,9,14]$.

Definition 2. A graph $G$ is $\left(H_{1}, \ldots, H_{r}\right)$-Ramsey-minimal if $G \rightarrow\left(H_{1}, \ldots, H_{r}\right)$, but for any proper subgraph $G^{\prime}$ of $G, G^{\prime} \nrightarrow\left(H_{1}, \ldots, H_{r}\right)$.

We define $\mathcal{R}_{\min }\left(H_{1}, \ldots, H_{r}\right)$ to be the family of all $\left(H_{1}, \ldots, H_{r}\right)$-Ramsey-minimal graphs. A graph $G$ is $\mathcal{R}_{\min }\left(H_{1}, \ldots, H_{r}\right)$-saturated if no element of $\mathcal{R}_{\min }\left(H_{1}, \ldots, H_{r}\right)$ is a subgraph of $G$, but for any edge $e$ in $\bar{G}$, some element of $\mathcal{R}_{\min }\left(H_{1}, \ldots, H_{r}\right)$ is a subgraph of $G+e$. It can be easily checked that a non-complete graph is $\left(H_{1}, \ldots, H_{r}\right)$-co-critical if and only if it is $\mathcal{R}_{\min }\left(H_{1}, \ldots, H_{r}\right)$-saturated. From now on, we shall use the notion of $\left(H_{1}, \ldots, H_{r}\right)$-co-critical other than $\mathcal{R}_{\min }\left(H_{1}, \ldots, H_{r}\right)$-saturated, as the former is much simpler and straightforward.

Let $R=R\left(K_{t_{1}}, \ldots, K_{t_{r}}\right)$ be the classical Ramsey number for $K_{t_{1}}, \ldots, K_{t_{r}}$. Hanson and Toft [12] proved that every $\left(K_{t_{1}}, \ldots, K_{t_{r}}\right)$-co-critical on $n$ vertices has at most $e\left(T_{R-1, n}\right)$ edges and this bound is best possible, where $T_{R-1, n}$ is the Turán graph on $n$ vertices without $K_{R}$. They also observed that for all $n \geqslant R$, the graph $K_{R-2}+\bar{K}_{n-R+2}$ is $\left(K_{t_{1}}, \ldots, K_{t_{r}}\right)$ co-critical. They further made the following conjecture that no $\left(K_{t_{1}}, \ldots, K_{t_{r}}\right)$-co-critical graph on $n$ vertices can have fewer than $e\left(K_{R-2}+\bar{K}_{n-R+2}\right)$ edges.

Conjecture 3 (Hanson and Toft [12]). Let $G$ be a $\left(K_{t_{1}}, \ldots, K_{t_{r}}\right)$-co-critical graph on $n$ vertices. Then

$$
e(G) \geqslant(R-2)(n-R+2)+\left(\begin{array}{c}
R-2 \\
2
\end{array}\right) .
$$

This bound is best possible for every $n$.

Conjecture 3 remains wide open, except that the first nontrivial case, $\left(K_{3}, K_{3}\right)$-cocritical graphs, has been settled in [5] for $n \geqslant 56$. Structural properties of $\left(K_{3}, K_{4}\right)$ co-critical graphs are given in [2]. Motivated by Conjecture 3, we study the following 
problem. Let $\mathcal{T}_{k}$ denote the family of all trees on $k$ vertices. For all $t, k \geqslant 3$, we write $G \rightarrow\left(K_{t}, \mathcal{T}_{k}\right)$ if for every 2-coloring $\tau: E(G) \rightarrow$ red, blue\}, $G$ has either a red $K_{t}$ or a blue tree $T_{k} \in \mathcal{T}_{k}$. A non-complete graph $G$ is $\left(K_{t}, \mathcal{T}_{k}\right)$-co-critical if $G \nrightarrow\left(K_{t}, \mathcal{T}_{k}\right)$, but $G+e \rightarrow\left(K_{t}, \mathcal{T}_{k}\right)$ for all $e$ in $\bar{G}$. The main purpose of this paper is to study the structural properties of $\left(K_{t}, \mathcal{T}_{k}\right)$-co-critical graphs on $n$ vertices in order to obtain the minimum size among all such graphs. By a classic result of Chvátal [4], $R\left(K_{t}, \mathcal{T}_{k}\right)=(t-1)(k-1)+1$. Hence, every $\left(K_{t}, \mathcal{T}_{k}\right)$-co-critical graph has at least $R\left(K_{t}, \mathcal{T}_{k}\right)=(t-1)(k-1)+1$ vertices. Following the observation made in both [10] and [12], every $\left(K_{t}, \mathcal{T}_{k}\right)$-co-critical graph on $n$ vertices has at most $e\left(T_{R\left(K_{t}, \mathcal{T}_{k}\right)-1, n}\right)$ edges. We focus on studying the minimum number of possible edges over all $\left(K_{t}, \mathcal{T}_{k}\right)$-co-critical graphs on $n$ vertices. Very recently, Rolek and the first author [14] proved the following.

Theorem 4 (Rolek and Song [14]). Let $n, k \in \mathbb{N}$.

(i) Every $\left(K_{3}, \mathcal{T}_{4}\right)$-co-critical graph on $n \geqslant 18$ vertices has at least $\lfloor 5 n / 2\rfloor$ edges. This bound is sharp for every $n \geqslant 18$.

(ii) For all $k \geqslant 5$, if $G$ is $\left(K_{3}, \mathcal{T}_{k}\right)$-co-critical on $n \geqslant 2 k+(\lceil k / 2\rceil+1)\lceil k / 2\rceil-2$ vertices, then

$$
e(G) \geqslant\left(\frac{3}{2}+\frac{1}{2}\left\lceil\frac{k}{2}\right\rceil\right) n-c(k)
$$

where $c(k)=\left(\frac{1}{2}\left\lceil\frac{k}{2}\right\rceil+\frac{3}{2}\right) k-2$. This bound is asymptotically best possible.

To state our results, we need to introduce more notation. Given a family $\mathcal{F}$, a graph is $\mathcal{F}$-free if it does not contain any graph $F \in \mathcal{F}$ as a subgraph. We simply say a graph is $F$-free when $\mathcal{F}=\{F\}$. Erdös, Hajnal and Moon [7] in 1964 initiated the study of the minimum number of edges over all $K_{t}$-saturated graphs on $n$ vertices (see the dynamic survey [8] on the extensive studies on $K_{t}$-saturated graphs). Theorem 5 below is a result of Day [6] on $K_{t}$-saturated graphs with prescribed minimum degree. It confirms a conjecture of Bollobás [1] when $t=3$. It is worth noting that Day applied $r$-neighbor bootstrap percolation on a $K_{t}$-saturated graph to prove Theorem 5 , where graph bootstrap percolation was introduced in [3]. Theorem 6 is a result of Hajnal [11] on $K_{t}$-saturated graphs.

Theorem 5 (Day [6]). Let $q \in \mathbb{N}$. There exists a constant $c=c(q)$ such that, for all $3 \leqslant t \in \mathbb{N}$ and all $n \in \mathbb{N}$, if $G$ is a $K_{t}$-saturated graph on $n$ vertices with $\delta(G) \geqslant q$, then $e(G) \geqslant q n-c$.

Theorem 6 (Hajnal [11]). Let $t, n \in \mathbb{N}$. Let $G$ be a $K_{t}$-saturated graph on $n$ vertices. Then either $\Delta(G)=n-1$ or $\delta(G) \geqslant 2(t-2)$. 
For a $\left(K_{t}, \mathcal{T}_{k}\right)$-co-critical graph $G$, let $\tau: E(G) \rightarrow\{$ red, blue $\}$ be a 2-coloring of $E(G)$ and let $E_{r}$ and $E_{b}$ be the color classes of the coloring $\tau$. We use $G_{r}$ and $G_{b}$ to denote the spanning subgraphs of $G$ with edge sets $E_{r}$ and $E_{b}$, respectively. We define $\tau$ to be a critical coloring of $G$ if $G$ has neither a red $K_{t}$ nor a blue $T_{k} \in \mathcal{T}_{k}$ under $\tau$, that is, if $G_{r}$ is $K_{t}$-free and $G_{b}$ is $\mathcal{T}_{k}$-free. For every $v \in V(G)$, we use $d_{r}(v)$ and $N_{r}(v)$ to denote the degree and neighborhood of $v$ in $G_{r}$, respectively. Similarly, we define $d_{b}(v)$ and $N_{b}(v)$ to be the degree and neighborhood of $v$ in $G_{b}$, respectively. One can see that if $G$ is $\left(K_{t}, \mathcal{T}_{k}\right)$-co-critical, then $G$ admits at least one critical coloring but $G+e$ admits no critical coloring for every edge $e$ in $\bar{G}$.

In this paper, we first establish a number of important structural properties of $\left(K_{t}, \mathcal{T}_{k}\right)$ co-critical graphs in the hope that the method we develop here may shed some light on attacking Conjecture 3. Theorem $7(\mathrm{~h})$ below is crucial in the proof of Theorem 8 . Following Day [6], we apply $q$-neighbor bootstrap percolation on a not necessarily $K_{t^{-}}$ saturated graph, to prove Theorem $7(\mathrm{~h})$, but with more involved rules.

Theorem 7. For all $t, k \in \mathbb{N}$ with $t \geqslant 3$ and $k \geqslant 3$, let $G$ be a $\left(K_{t}, \mathcal{T}_{k}\right)$-co-critical graph on $n$ vertices. Among all critical colorings of $G$, let $\tau: E(G) \rightarrow\{$ red, blue $\}$ be a critical coloring of $G$ with $\left|E_{r}\right|$ maximum. Let $D_{1}, \ldots, D_{p}$ be all components of $G_{b}$. Let $H:=G \backslash\left(\bigcup_{i \in[p]} E\left(G\left[V\left(D_{i}\right)\right]\right)\right)$. Then the following hold.

(a) $\Delta\left(G_{r}\right) \leqslant n-2$ and $\delta\left(G_{r}\right) \geqslant 2(t-2)$.

(b) For all $i, j \in[p]$ with $i \neq j$, if there exist $u \in V\left(D_{i}\right)$ and $v \in V\left(D_{j}\right)$ such that $u v \notin E(H)$, then $H\left[N_{H}(u) \cap N_{H}(v)\right]$ contains $K_{t-2}$ as a subgraph.

(c) For every $u v \in E(H)$, if $v$ is contained in all $K_{t-2}$ subgraphs of $H\left[N_{H}(u)\right]$ and $\{v\}=V\left(D_{j}\right)$ for some $j \in[p]$, then $\left|D_{i}\right|=k-1$ for all $D_{i}$ with $u \notin D_{i}$ and $D_{i} \backslash N_{H}(u) \neq \varnothing$, where $i \in[p]$.

(d) If $\delta(H) \leqslant 2 t-5$ and $k \geqslant t$, then for any vertex $u \in V(H)$ with $d_{H}(u)=\delta(H)$, no edge of $H\left[N_{H}(u)\right]$ is contained in all $K_{t-2}$ subgraphs of $H\left[N_{H}(u)\right]$.

(e) $k \geqslant 2 t-1-\delta(H)$ and $\delta(H) \geqslant t-1$.

(f) $\sum_{i=1}^{p} e_{G}\left(V\left(D_{i}\right)\right)>\left(\frac{1}{2}\left\lceil\frac{k}{2}\right\rceil-\frac{1}{2}\right)(n-(t-1)(\lceil k / 2\rceil-1))$.

(g) $H$ is connected.

(h) For every $q \in \mathbb{N}$ with $q \geqslant t-1$, there exists a constant $c(q, k)$ such that, if $\delta(H) \geqslant q$, then $e(H) \geqslant q n-c(q, k)$. 
We prove Theorem 7 in Section 2. We then apply Theorem 7 to study the size of $\left(K_{t}, \mathcal{T}_{k}\right)$-co-critical graphs. We prove Theorem 8 in Section 3.

Theorem 8. Let $t, k \in \mathbb{N}$ with $t \geqslant 4$ and $k \geqslant \max \{6, t\}$. There exists a constant $\ell(t, k)$ such that, for all $n \in \mathbb{N}$ with $n \geqslant(t-1)(k-1)+1$, if $G$ is a $\left(K_{t}, \mathcal{T}_{k}\right)$-co-critical graph on $n$ vertices, then

$$
e(G) \geqslant\left(\frac{4 t-9}{2}+\frac{1}{2}\left\lceil\frac{k}{2}\right\rceil\right) n-\ell(t, k) .
$$

Finally we prove that the linear bound given in Theorem 8 is asymptotically best possible when $t \in\{4,5\}$ and $k \geqslant 6$. Proof of Theorem 9 is given in Section 4 .

Theorem 9. For each $t \in\{4,5\}$, all $k \geqslant 3$ and $n \geqslant(2 t-3)(k-1)+\lceil k / 2\rceil\lceil k / 2\rceil-1$, there exists a $\left(K_{t}, \mathcal{T}_{k}\right)$-co-critical graph $G$ on $n$ vertices such that

$$
e(G) \leqslant\left(\frac{4 t-9}{2}+\frac{1}{2}\left\lceil\frac{k}{2}\right\rceil\right) n+C(t, k)
$$

where $C(t, k)=\frac{1}{2}\left(t^{2}+t-5\right) k^{2}-\left(2 t^{2}+2 t-11\right) k-\frac{(t-2)(t-19)}{2}-\frac{1}{2}\left\lceil\frac{k}{2}\right\rceil\left((2 t-3)(k-1)-\left\lceil\frac{k}{2}\right\rceil\right)$ when $k \geqslant 4$ and $C(t, 3)=-2 t^{2}+5 t-2$.

With the support of Theorem 4 and Theorem 9, we believe that the linear bound given in Theorem 8 is asymptotically best possible for all $t \geqslant 3$ and $k \geqslant 3$.

\section{Structural properties of $\left(K_{t}, \mathcal{T}_{k}\right)$-co-critical graphs}

We first prove the following lemma.

Lemma 10. For all $t, k \in \mathbb{N}$ with $t \geqslant 3$ and $k \geqslant 3$, let $G$ be a $\left(K_{t}, \mathcal{T}_{k}\right)$-co-critical graph on $n$ vertices. Let $\tau: E(G) \rightarrow\{$ red, blue $\}$ be a critical coloring of $G$. Then the following hold.

(a) For every component $D$ of $G_{b},|D| \leqslant k-1$ and $G[V(D)]=K_{|D|}$.

(b) If $D_{1}, \cdots, D_{q}$ are the components of $G_{b}$ with $\left|D_{i}\right|<k / 2$ for all $i \in[q]$, then $V\left(D_{1}\right)$, $\cdots, V\left(D_{q}\right)$ are complete to each other in $G_{r}$, and so $q \leqslant t-1$.

Proof. To prove (a), let $D$ be a component of $G_{b}$. Since $G_{b}$ is $\mathcal{T}_{k}$-free, we see that $|D| \leqslant k-1$. Suppose next that $G[V(D)] \neq K_{|D|}$. Let $u, v \in V(D)$ be such that $u v \notin E(G)$. We obtain a critical coloring of $G+u v$ from $\tau$ by coloring the edge $u v$ blue, a contradiction.

To prove (b), suppose there exist vertices $u \in V\left(D_{i}\right)$ and $v \in V\left(D_{j}\right)$ such that $u v \notin E_{r}$, where $i, j \in[q]$ with $i \neq j$. Then $u v \notin E(G)$ and so we obtain a critical coloring of $G+u v$ from $\tau$ by coloring the edge $u v$ blue, a contradiction. Thus $V\left(D_{1}\right), \cdots, V\left(D_{q}\right)$ are complete to each other in $G_{r}$. Since $G_{r}$ is $K_{t}$-free, we have $q \leqslant t-1$. 
We are now ready to prove Theorem 7 .

Proof of Theorem 7: Let $G, \tau, D_{1}, \ldots, D_{p}$ and $H$ be given as in the statement. Then $n \geqslant(t-1)(k-1)+1$. By Lemma 10(a), $\left|D_{i}\right| \leqslant k-1$ for all $i \in[p]$. Hence, $G_{b}$ has at least $t$ components because $\left|G_{b}\right|=n \geqslant(t-1)(k-1)+1$. We first prove Theorem 7 (a). Since $\tau$ was chosen so that $\left|E_{r}\right|$ is maximum, $G_{r}$ is $K_{t}$-free but $G_{r}+e$ contains a copy of $K_{t}$ for every $e \in E\left(\overline{G_{r}}\right)$. Hence $G_{r}$ is $K_{t}$-saturated. Suppose there exists a vertex $x \in V(G)$ such that $d_{r}(x)=n-1$. Note that $G_{r} \backslash x$ is $K_{t-1}$-free because $G_{r}$ is $K_{t}$-free. Since $G \neq K_{n}$, there must exist $u, w \in N_{r}(x)$ such that $u w \notin E(G)$. By Lemma 10(a), $u, w$ belong to different components of $G_{b}$. But then we obtain a critical coloring of $G+u w$ from $\tau$ by first coloring the edge $u w$ red, and then recoloring $x u$ blue and all edges incident with $u$ in $G_{b}$ red, contrary to the fact that $G+u w$ has no critical coloring. This proves that $\Delta\left(G_{r}\right) \leqslant n-2$. Since $G_{r}$ is $K_{t}$-saturated, by Theorem $6, \delta\left(G_{r}\right) \geqslant 2(t-2)$.

To prove Theorem $7(\mathrm{~b})$, let $u \in V\left(D_{i}\right)$ and $v \in V\left(D_{j}\right)$ be such that $u v \notin E(H)$, where $i \neq j$. Suppose $H\left[N_{H}(u) \cap N_{H}(v)\right]$ is $K_{t-2}$-free. Since $\left|D_{\ell}\right| \leqslant k-1$ for all $\ell \in[p]$, we obtain a critical coloring of $G+u v$ from $\tau$ by first coloring the edge $u v$ red, and then recoloring all red edges in $G\left[V\left(D_{\ell}\right)\right]$ blue for all $\ell \in[p]$, a contradiction. Therefore, $H\left[N_{H}(u) \cap N_{H}(v)\right]$ contains $K_{t-2}$ as a subgraph. This proves Theorem $7(\mathrm{~b})$.

To prove Theorem $7(\mathrm{c})$, let $u v \in E(H)$ be such that $v$ is contained in all $K_{t-2}$ subgraphs of $H\left[N_{H}(u)\right]$ and $\{v\}=V\left(D_{j}\right)$ for some $j \in[p]$. We may assume that $u \in V\left(D_{p}\right)$ and $\{v\}=V\left(D_{p-1}\right)$. Note that $H\left[N_{H}(u)\right] \backslash v$ is $K_{t-2}$-free. Suppose there exists an $\ell \in[p-2]$ such that $D_{\ell} \backslash N_{H}(u) \neq \varnothing$ but $\left|D_{\ell}\right| \leqslant k-2$. Let $w \in V\left(D_{\ell}\right) \backslash N_{H}(u)$. Then $w v \in E_{r}$, else we obtain a critical coloring of $G+w v$ from $\tau$ by coloring the edge $w v$ blue. Since $H\left[N_{H}(u)\right] \backslash v$ is $K_{t-2}$-free, we then obtain a critical coloring of $G+u w$ from $\tau$ by coloring the edge $u w$ red, and then recoloring $w v$ blue and all red edges incident with $u$ in $G\left[V\left(D_{p}\right)\right]$ blue, a contradiction. This proves Theorem $7(\mathrm{c})$.

To prove Theorem $7(\mathrm{~d}, \mathrm{e})$, let $u \in V(H)$ with $d_{H}(u)=\delta(H)$. We may assume that $u \in$ $V\left(D_{p}\right)$. By Theorem $7(\mathrm{~b}), d_{H}(u) \geqslant t-2$. Let $N_{H}(u):=\left\{u_{1}, \ldots, u_{\delta(H)}\right\}$. By Theorem $7(\mathrm{~b})$ applied to $u$ and any vertex in $V(H) \backslash\left(V\left(D_{p}\right) \cup N_{H}(u)\right)$, we see that $H\left[N_{H}(u)\right]$ must contain $K_{t-2}$ as a subgraph. We may assume that $H\left[\left\{u_{1}, \ldots, u_{t-2}\right\}\right]=K_{t-2}$. Then we may further assume that $u_{i} \in V\left(D_{p-i}\right)$ for all $i \in[t-2]$. Let $v \in V(H) \backslash\left(V\left(D_{p}\right) \cup N_{H}(u)\right)$.

To proceed to prove Theorem $7(\mathrm{~d})$, assume $d_{H}(u) \leqslant 2 t-5$ and $k \geqslant t$. Suppose $H\left[N_{H}(u)\right]$ has an edge, say $u_{1} u_{2}$, that is contained in all $K_{t-2}$ subgraphs of $H\left[N_{H}(u)\right]$. Then both $H\left[N_{H}(u) \backslash u_{1}\right]$ and $H\left[N_{H}(u) \backslash u_{2}\right]$ are $K_{t-2}$-free. By Theorem $7(\mathrm{~b})$ applied to $u$ and any vertex in $V(H) \backslash\left(V\left(D_{p}\right) \cup N_{H}(u)\right), V(H) \backslash\left(V\left(D_{p}\right) \cup N_{H}(u)\right)$ must be complete to $\left\{u_{1}, u_{2}\right\}$ in $H$. Then $V\left(D_{p-1}\right) \cup V\left(D_{p-2}\right) \subseteq N_{H}(u) \backslash\left\{u_{3}, \ldots, u_{t-2}\right\}$. Thus $\left|V\left(D_{p-1}\right) \cup V\left(D_{p-2}\right)\right|=\delta(H)-(t-4) \leqslant t-1 \leqslant k-1$, because $\delta(H) \leqslant 2 t-5$ and $t \leqslant k$. Then we obtain a critical coloring of $G+u v$ from $\tau$ by first coloring the edge $u v$ 
red, and then recoloring $u_{1} u_{2}$ blue and all red edges incident with $u$ in $G\left[V\left(D_{p}\right)\right]$ blue, a contradiction. This proves Theorem $7(\mathrm{~d})$.

To proceed to prove Theorem $7(\mathrm{e})$, note that $\left|N_{r}(u) \cap V\left(D_{p}\right)\right|=\left|N_{r}(u)\right|-d_{H}(u)$. By Theorem $7(\mathrm{a}),\left|N_{r}(u)\right| \geqslant 2 t-4$. Since $D_{p}$ is a component of $G_{b}$, we see that $N_{b}(u) \cap$ $V\left(D_{p}\right) \neq \varnothing$. It follows that $\left|V\left(D_{p}\right)\right|=|\{u\}|+\left|N_{b}(u) \cap V\left(D_{p}\right)\right|+\left|N_{r}(u) \cap V\left(D_{p}\right)\right| \geqslant$ $1+1+(2 t-4)-d_{H}(u)=2 t-2-d_{H}(u)$. By Lemma 10(a), $2 t-2-d_{H}(u) \leqslant\left|V\left(D_{p}\right)\right| \leqslant k-1$, which yields $k \geqslant 2 t-1-d_{H}(u)$. Suppose next that $\delta(H)=t-2<2 t-5$. Then $k \geqslant t+1$. But then $H\left[\left\{u_{1}, \ldots, u_{t-2}\right\}\right]$ is the only $K_{t-2}$ subgraph of $H\left[N_{H}(u)\right]$, contrary to Theorem $7(\mathrm{~d})$. This proves Theorem $7(\mathrm{e})$.

We next prove Theorem 7 (f). By Lemma $10(\mathrm{a}, \mathrm{b}),\left|D_{i}\right| \leqslant k-1, G\left[V\left(D_{i}\right)\right]=K_{\left|D_{i}\right|}$ for all $i \in[p]$, and at most $t-1$ of the $D_{i}$ 's have less than $k / 2$ vertices. Since $n \geqslant(t-1)(k-1)+1$, we see that $n-(t-2)(\lceil k / 2\rceil-1) \geqslant(t-2)\lfloor k / 2\rfloor+k$. Let $r$ be the remainder of $n-(t-2)(\lceil k / 2\rceil-1)$ when divided by $\lceil k / 2\rceil$, and let $s \geqslant 0$ be an integer such that

$$
n-(t-2)(\lceil k / 2\rceil-1)=s\lceil k / 2\rceil+r .
$$

Then $0 \leqslant r \leqslant\lceil k / 2\rceil-1$. Let $\ell=t-2+\min \{1, r\}$. It is straightforward to see that if $r \neq 0$, then $\ell=t-1$ and $\sum_{i=1}^{p} e_{G}\left(V\left(D_{i}\right)\right)$ is minimized when: $t-1$ of the components, say $D_{1}, \ldots, D_{\ell}$ are such that $\left|D_{1}\right|, \ldots,\left|D_{t-1}\right|<k / 2$ with $\left|D_{1}\right|+\cdots+\left|D_{t-1}\right|=$ $(t-2)(\lceil k / 2\rceil-1)+r$, and the remaining $s$ components, $D_{t}, \cdots, D_{t-1+s}$ are such that $\left|D_{t}\right|=$ $\cdots=\left|D_{t-1+s}\right|=\lceil k / 2\rceil$; if $r=0$, then $\ell=t-2$ and $\sum_{i=1}^{p} e_{G}\left(V\left(D_{i}\right)\right)$ is minimized when: $t-1$ of the components, say $D_{1}, D_{2}, \ldots, D_{t-1}$ are such that $\left|D_{1}\right|, \ldots,\left|D_{t-1}\right|<k / 2$ with $\left|D_{1}\right|+\cdots+\left|D_{t-1}\right|=(t-2)(\lceil k / 2\rceil-1)$, and the remaining $s$ components, $D_{t}, \cdots, D_{t-1+s}$ are such that $\left|D_{t}\right|=\cdots=\left|D_{t-1+s}\right|=\lceil k / 2\rceil$. Using the facts that $s\lceil k / 2\rceil+r=n-(t-$ $2)(\lceil k / 2\rceil-1)$ and $r \leqslant\lceil k / 2\rceil-1$, it follows that

$$
\begin{aligned}
\sum_{i=1}^{p} e_{G}\left(V\left(D_{i}\right)\right)>s\left(\begin{array}{c}
\lceil/ 2\rceil \\
2
\end{array}\right) & =\left(s \cdot\left\lceil\frac{k}{2}\right\rceil\right)\left(\frac{1}{2}\left\lceil\frac{k}{2}\right\rceil-\frac{1}{2}\right) \\
& \geqslant(n-(t-1)(\lceil k / 2\rceil-1))\left(\frac{1}{2}\left\lceil\frac{k}{2}\right\rceil-\frac{1}{2}\right) \\
& =\left(\frac{1}{2}\left\lceil\frac{k}{2}\right\rceil-\frac{1}{2}\right)(n-(t-1)(\lceil k / 2\rceil-1)) .
\end{aligned}
$$

This proves Theorem $7(\mathrm{f})$.

To prove Theorem $7(\mathrm{~g})$, suppose that $H$ is disconnected. Let $x, y \in V(H)$ be such that $x$ and $y$ are in different components of $H$. By Theorem $7(\mathrm{~b}),\{x, y\} \subseteq D_{i}$ for some $i \in[p]$, and there must exist a vertex $w \in D_{j}$ such that $x w \notin E(H)$ and $y w \in E(H)$, where $j \in[p]$ with $j \neq i$. By Theorem $7(\mathrm{~b}), x$ and $w$ have at least $t-2$ common neighbors in $H$. But then $x$ and $y$ must be in the same component of $H$, a contradiction. This proves Theorem $7(\mathrm{~g})$. 
It remains to prove Theorem $7(\mathrm{~h})$. By Theorem $7(\mathrm{~g}), H$ is connected. Let $q \in \mathbb{N}$ with $q \geqslant t-1$. Assume $\delta(H) \geqslant q$. Following Day [6], we next apply $q$-neighbor bootstrap percolation on $H$. Note that $H$ is not necessarily $K_{t}$-saturated. Given a set $S \subseteq V(H)$ and any vertex $v \in V(H)$, let $N_{S}(v):=N_{H}(v) \cap S$ and $d_{S}(v):=\left|N_{S}(v)\right|$. Let $R \subseteq V(H)$ be any nonempty set. Let $R^{0}:=R$ and for $i \geqslant 1$, let

$$
R^{i}:=R^{i-1} \cup\left\{v \in V(H) \mid d_{R^{i-1}}(v) \geqslant q\right\} .
$$

Let $\bar{R}:=\bigcup_{i \geqslant 0} R^{i}$, the closure of $R$ under the $q$-neighbor bootstrap percolation on $H$. Then

$$
e(H[\bar{R}]) \geqslant q(|\bar{R}|-|R|),
$$

because every vertex in $R^{i} \backslash R^{i-1}$ is adjacent to at least $q$ vertices in $R^{i-1}$. Let $Y(R):=$ $V(H) \backslash \bar{R}$. Finally, for any $v \in V(H)$, let

$$
\omega_{R}(v):=d_{\bar{R}}(v)+d_{Y(R)}(v) / 2 .
$$

We call $\omega_{R}(v)$ the weight of $v$ (with respect to $R$ ). Then

$$
e_{H}(\bar{R}, Y(R))+e_{H}(Y(R))=\sum_{v \in Y(R)} \omega_{R}(v) .
$$

Within $Y(R)$, we define $B(R)$ to be the set $\left\{v \in Y(R) \mid \omega_{R}(v)<q\right\}$, which we call the set of bad vertices. We next show that there exists a constant $c_{1}(q, k)$ and a nonempty set $R \subseteq V(H)$ with $|R| \leqslant c_{1}(q, k)$ such that $B(R)=\varnothing$.

Assume $B(R) \neq \varnothing$ for our initial $R$. Our goal is to move a small number of vertices into $R$ so that the remaining vertices in $B(R)$ have strictly larger weight. To achieve this, let

$$
\mathcal{U}_{R}:=\left\{U \subseteq R \mid U=N_{R}(v) \text { for some } v \in B(R)\right\} .
$$

Note that for every vertex $v \in B(R), d_{R}(v) \leqslant q-1$. Thus

$$
\left|\mathcal{U}_{R}\right| \leqslant 1+|R|+\left(\begin{array}{c}
|R| \\
2
\end{array}\right)+\cdots+\left(\begin{array}{c}
|R| \\
q-1
\end{array}\right) .
$$

Let $\mathcal{U}_{R}:=\left\{U_{1}, U_{2}, \ldots, U_{\left|\mathcal{U}_{R}\right|}\right\}$ and let $u_{i} \in B(R)$ with $N_{R}\left(u_{i}\right)=U_{i}$ for all $i \in\left\{1, \ldots,\left|\mathcal{U}_{R}\right|\right\}$. Then $d_{\bar{R}}\left(u_{i}\right)<q$, and so $d_{Y(R)}\left(u_{i}\right) \geqslant 1$ because $d_{H}\left(u_{i}\right) \geqslant q$. Let $x_{i} \in Y(R)$ such that $u_{i} x_{i} \in E(H)$ for all $i \in\left\{1, \ldots,\left|\mathcal{U}_{R}\right|\right\}$, and let $X(R):=\left\{x_{1}, x_{2}, \ldots, x_{\left|\mathcal{U}_{R}\right|}\right\}$. By the choice of $\mathcal{U}_{R}$ and $u_{1}, u_{2}, \ldots, u_{\left|\mathcal{u}_{R}\right|}$, for every vertex $v \in B(R)$, we see that $N_{R}(v)=N_{R}\left(u_{i}\right)$ for some $i \in\left\{1,2, \ldots,\left|\mathcal{U}_{R}\right|\right\}$. Finally, let

$S(R):=\left\{v \in B(R) \mid N_{R}(v)=N_{R}\left(u_{i}\right)\right.$ and $\left\{v, x_{i}\right\} \subseteq D_{j}$ for some $i \in\left[\left|\mathcal{U}_{R}\right|\right]$ and $\left.j \in[p]\right\}$.

We next show that Algorithm 1 below yields a nonempty set $R \subseteq V(H)$ with $B(R)=\varnothing$. 


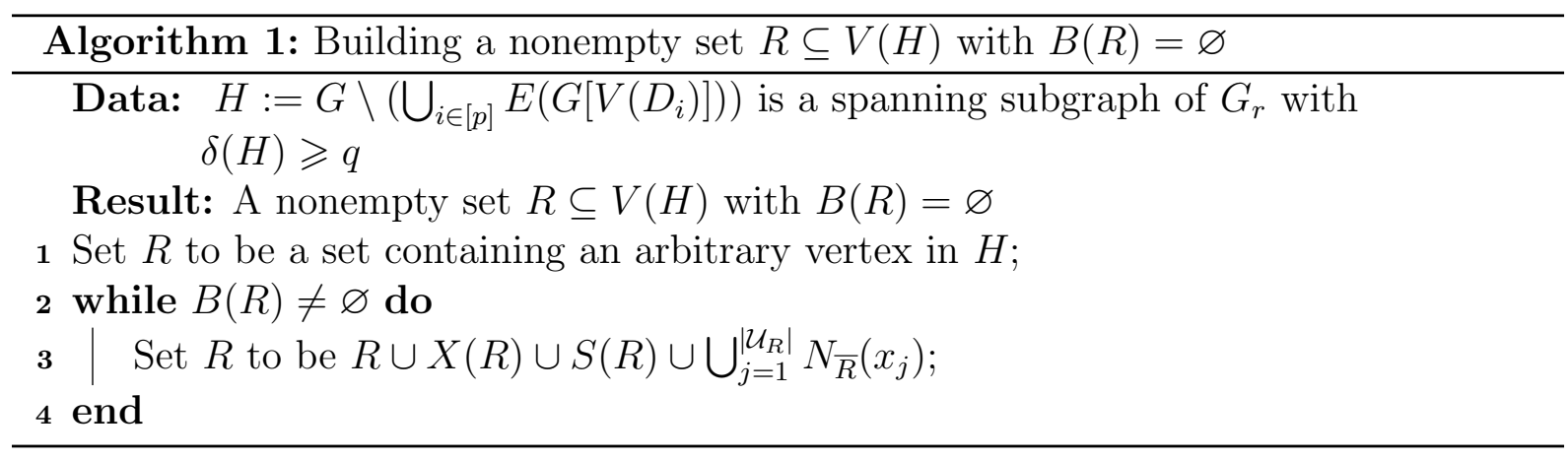

Let $R_{i}$ be the set $R$ obtained in the $i$-th iteration of Line 2 when running Algorithm 1 . Then for all $i \geqslant 1, R_{i-1} \subseteq R_{i}, \bar{R}_{i-1} \subseteq \bar{R}_{i}, Y\left(R_{i}\right) \subseteq Y\left(R_{i-1}\right)$ and $B\left(R_{i}\right) \subseteq B\left(R_{i-1}\right)$. To see why $\omega_{R_{i}}(v) \geqslant \omega_{R_{i-1}}(v)$ for all $v \in B\left(R_{i}\right)$, we next introduce a control function on $V(H)$, because dealing with $\omega_{R}(v)$ directly is difficult. Let $\phi_{R}(v):=\sum_{x \in N_{H}(v)} f_{R}(x)$ for all $v \in V(H)$, where for all $x \in V(H)$,

$$
f_{R}(x)= \begin{cases}1, & \text { if } x \in R \\ 1 / 2, & \text { if } x \in \bar{R} \backslash R \\ d_{R}(x) /(2 q), & \text { if } x \in Y(R)\end{cases}
$$

It is worth noting that $\phi_{R}(v) \leqslant \omega_{R}(v)$ for every vertex $v \in V(H)$, because $d_{R}(x) \leqslant q-1$ for all $x \in Y(R)$. Similarly, for all $i \geqslant 1, f_{R_{i-1}}(x) \leqslant f_{R_{i}}(x)$ for every $x \in V(H)$, because $Y\left(R_{i}\right) \subseteq Y\left(R_{i-1}\right)$. We next claim that

$(*)$ for all $i \geqslant 1$ and every $v \in B\left(R_{i}\right), \phi_{R_{i}}(v) \geqslant \phi_{R_{i-1}}(v)+1 /(2 q)$.

Proof. Let $i \geqslant 1$ and $v \in B\left(R_{i}\right)$. Then $v \in B\left(R_{i-1}\right)$, since $B\left(R_{i}\right) \subseteq B\left(R_{i-1}\right)$. Let $\mathcal{U}_{R_{i-1}}$, $\left\{u_{1}, \ldots, u_{\left|\mathcal{U}_{R_{i-1}}\right|}\right\} \subseteq B\left(R_{i-1}\right)$, and $\left\{x_{1}, \ldots, x_{\left|\mathcal{U}_{R_{i-1}}\right|}\right\} \subseteq Y\left(R_{i-1}\right)$ be defined accordingly for $R_{i-1}$. Then $N_{R_{i-1}}(v)=N_{R_{i-1}}\left(u_{j}\right)$ for some $j \in\left\{1,2, \ldots,\left|\mathcal{U}_{R_{i-1}}\right|\right\}$. To prove $\phi_{R_{i}}(v) \geqslant$ $\phi_{R_{i-1}}(v)+1 /(2 q)$, it suffices to show that $f_{R_{i}}(x) \geqslant f_{R_{i-1}}(x)+1 /(2 q)$ for some $x \in N_{H}(v)$. Since $\left\{x_{1}, \ldots, x_{\left|\mathcal{U}_{R_{i-1}}\right|}\right\} \subseteq Y\left(R_{i-1}\right) \cap R_{i}$, we see that $f_{R_{i-1}}(x)=d_{R_{i-1}}(x) /(2 q) \leqslant(q-$ $1) /(2 q)=1 / 2-1 /(2 q)$, and $f_{R_{i}}(x)=1>f_{R_{i-1}}(x)+1 /(2 q)$ for all $x \in\left\{x_{1}, \ldots, x_{\left.\mid \mathcal{U}_{R_{i-1}}\right\}}\right\}$. We may assume that $v x_{\ell} \notin E(H)$ for all $\ell \in\left\{1, \ldots,\left|\mathcal{U}_{R_{i-1}}\right|\right\}$, otherwise we are done. Since $v \in B\left(R_{i}\right)$, by the choice of $x_{j}$ and $S\left(R_{i-1}\right)$, we see that $\left\{v, x_{j}\right\} \nsubseteq V\left(D_{\ell}\right)$ for all $\ell \in[p]$. By Theorem $7(\mathrm{~b})$ applied to $v$ and $x_{j}, H\left[N_{H}(v) \cap N_{H}\left(x_{j}\right)\right]$ contains $K_{t-2}$ as a subgraph. Let $W$ be the vertex set of such a $K_{t-2}$ subgraph. It follows that $W \nsubseteq R_{i-1}$, else $G_{r}\left[W \cup\left\{u_{j}, x_{j}\right\}\right]=K_{t}$, since $N_{R_{i-1}}(v)=N_{R_{i-1}}\left(u_{j}\right)$ and $u_{j} x_{j} \in E(H)$. Let $x \in W \backslash R_{i-1}$.

If $x \in \bar{R}_{i-1} \backslash R_{i-1}$, then $f_{R_{i-1}}(x)=1 / 2$ and $f_{R_{i}}(x)=1$, and so $f_{R_{i}}(x) \geqslant f_{R_{i-1}}(x)+$ $1 /(2 q)$, as desired. If $x \in Y\left(R_{i-1}\right)$, then either $x \in \bar{R}_{i}$ or $x \in Y\left(R_{i}\right)$. In both cases, we have $f_{R_{i-1}}(x)=d_{R_{i-1}}(x) /(2 q) \leqslant 1 / 2-1 /(2 q)$. If $x \in \bar{R}_{i}$, then $f_{R_{i}}(x) \geqslant 1 / 2$ and so $f_{R_{i}}(x) \geqslant f_{R_{i-1}}(x)+1 /(2 q)$. Finally, if $x \in Y\left(R_{i}\right)$, then $d_{R_{i}}(x) \geqslant d_{R_{i-1}}(x)+1$ because 
$x_{j} \in R_{i} \backslash R_{i-1}$ and $R_{i-1} \subseteq R_{i}$. Hence, $f_{R_{i}}(x)=d_{R_{i}}(x) /(2 q) \geqslant\left(d_{R_{i-1}}(x)+1\right) /(2 q)=$ $f_{R_{i-1}}(x)+1 /(2 q)$.

In all cases, we have shown that there exists some vertex $x \in N_{H}(v)$ such that $f_{R_{i}}(x) \geqslant$ $f_{R_{i-1}}(x)+1 /(2 q)$. Hence, $\phi_{R_{i}}(v) \geqslant \phi_{R_{i-1}}(v)+1 /(2 q)$ for all $i \geqslant 1$ and $v \in B\left(R_{i}\right)$.

By $(*)$, Algorithm 1 stops after $m \leqslant 2 q^{2}$ iterations of Line 2 because $\phi_{R}(v) \leqslant \omega_{R}(v)<q$ for each $v \in B(R)$. Hence $R_{m} \subseteq V(H)$ with $R_{m} \neq \varnothing$ but $B\left(R_{m}\right)=\varnothing$. For all $i \geqslant 0$,

$$
\begin{aligned}
\left|R_{i+1}\right| & \leqslant\left|R_{i}\right|+\left|X\left(R_{i}\right)\right|+\left|S\left(R_{i}\right)\right|+\left|\bigcup_{j=1}^{\left|\mathcal{U}_{R_{i}}\right|} N_{\bar{R}_{i}}\left(x_{j}\right)\right| \\
& \leqslant\left|R_{i}\right|+\left|\mathcal{U}_{R_{i}}\right|+(k-2)\left|\mathcal{U}_{R_{i}}\right|+(q-1)\left|\mathcal{U}_{R_{i}}\right| \\
& =\left|R_{i}\right|+(k+q-2)\left|\mathcal{U}_{R_{i}}\right| \\
& \leqslant\left|R_{i}\right|+(k+q-2)\left(1+\left|R_{i}\right|+\left(\begin{array}{c}
\left|R_{i}\right| \\
2
\end{array}\right)+\cdots+\left(\begin{array}{c}
\left|R_{i}\right| \\
q-1
\end{array}\right)\right),
\end{aligned}
$$

which only depends on $q$ and $k$. It follows that by Algorithm 1, there exists a constant $c_{1}(q, k)$ and a non-empty set $R \subseteq V(H)$ with $|R| \leqslant c_{1}(q, k)$ such that $B(R)=\varnothing$. Then $\omega_{R}(v) \geqslant q$ for all $v \in Y(R)$ and so

$$
e_{H}(\bar{R}, Y(R))+e_{H}(Y(R))=\sum_{v \in Y(R)} \omega_{R}(v) \geqslant q|Y(R)|
$$

Therefore,

$$
\begin{aligned}
e(H) & =e(H[\bar{R}])+e_{H}(\bar{R}, Y(R))+e_{H}(Y(R)) \\
& \geqslant q(|\bar{R}|-|R|)+q|Y(R)| \\
& \geqslant q\left(|\bar{R}|-c_{1}(q, k)\right)+q|Y(R)| \\
& =q\left(n-c_{1}(q, k)\right) \\
& =q n-c(q, k)
\end{aligned}
$$

where $c(q, k)=q c_{1}(q, k)$. This proves Theorem $7(\mathrm{~h})$.

This completes the proof of Theorem 7 .

\section{Lower bound on the size of $\left(K_{t}, \mathcal{T}_{k}\right)$-co-critical graphs}

We begin this section with a useful lemma, which may be of independent interest. We use $\alpha(G)$ and $\omega(G)$ to denote the independence number and clique number of $G$, respectively. It is worth noting that Lemma 12 is stronger than Theorem 11 when $\alpha(G)>|G| / 2$ : Theorem 11 yields that $\left|\bigcap_{S \in \mathcal{F}} S\right|>0$, while Lemma 12 not only yields $\left|\bigcap_{S \in \mathcal{F}} S\right| \geqslant \delta(G)+1$ but also characterizes the case when $\left|\bigcap_{S \in \mathcal{F}} S\right|=1$. For completeness, we include a proof here due to Hehui Wu. For a graph $G$, a set $A \subseteq V(G)$ is stable if $G[A]$ has no edges. 
Theorem 11 (Hajnal [11]). Let $G$ be a graph and let $\mathcal{F}$ be the family of all maximum stable sets of $G$. Then

$$
\left|\bigcap_{S \in \mathcal{F}} S\right|+\left|\bigcup_{S \in \mathcal{F}} S\right| \geqslant 2 \alpha(G) .
$$

Lemma 12. Let $G$ be a graph with $\alpha(G)>|G| / 2$ and let $\mathcal{F}$ be the family of all maximum stable sets of $G$. Then

$$
\left|\bigcap_{S \in \mathcal{F}} S\right| \geqslant \delta(G)+2 \alpha(G)-|G| \geqslant \delta(G)+1 .
$$

Moreover, if $\bigcap_{S \in \mathcal{F}} S=\{u\}$, then $\alpha(G)=(|G|+1) / 2$ and $u$ is an isolated vertex in $G$.

Proof. Let $X \in \mathcal{F}$ and $Y:=V(G) \backslash X$. Then $|X|=\alpha(G)>|G| / 2$, and so $|X|>|Y|$. Let $H:=G[X, Y]$ be the bipartite subgraph of $G$ with $V(H)=X \cup Y$ and $E(H)=\{x y \in$ $E(G): x \in X, y \in Y\}$. Let $T$ be a stable set of $H$ with $|T|$ maximum and let $X_{1}:=X \backslash T$, $Y_{1}:=Y \cap T$ and $Y_{2}:=Y \backslash T$. Then $\left|Y_{1}\right|+\left|X \backslash X_{1}\right|=|T| \geqslant|X|=\left|X_{1}\right|+\left|X \backslash X_{1}\right|>$ $|Y|=\left|Y_{1}\right|+\left|Y_{2}\right|$, which implies that $\left|X_{1}\right| \leqslant\left|Y_{1}\right|$ and $\left|X \backslash X_{1}\right|>\left|Y_{2}\right|$. We next show that $H^{\prime}:=G\left[X \backslash X_{1}, Y_{2}\right]$ contains a matching that saturates $Y_{2}$. For any $S \subseteq Y_{2}$, we have $\left|N_{H^{\prime}}(S)\right| \geqslant|S|$, else $T^{\prime}:=\left(T \backslash N_{H^{\prime}}(S)\right) \cup S$ is a stable set of $H$ with $\left|T^{\prime}\right|>|T|$, a contradiction. By Hall's Theorem, there exists a matching, say $M$, of $H^{\prime}$ that saturates $Y_{2}$. Let $X_{2}:=V(M) \cap X$ and $X_{3}:=X \backslash\left(X_{1} \cup X_{2}\right)$. Then

$$
\left|X_{3}\right|=|X|-\left|X_{1}\right|-\left|X_{2}\right| \geqslant|X|-|Y|=2 \alpha(G)-|G|>0
$$

because $\left|X_{1}\right| \leqslant\left|Y_{1}\right|,\left|X_{2}\right|=\left|Y_{2}\right|$ and $\alpha(G)>|G| / 2$. Note that $X_{1} \cup Y_{1}$ is anti-complete to $X \backslash X_{1}$ in $H$. By the choice of $T, \alpha\left(H\left[X_{1} \cup Y_{1}\right]\right) \leqslant\left|X_{1}\right|$. Moreover, $\alpha\left(H\left[X_{2} \cup Y_{2}\right]\right) \leqslant\left|X_{2}\right|$ because $M$ is a perfect matching of $G\left[X_{2}, Y_{2}\right]$. Then for any $S \in \mathcal{F},\left|S \cap\left(X_{1} \cup Y_{1}\right)\right| \leqslant\left|X_{1}\right|$ and $\left|S \cap\left(X_{2} \cup Y_{2}\right)\right| \leqslant\left|X_{2}\right|$. Therefore, $\left|X_{3}\right| \geqslant\left|S \cap X_{3}\right|=|S|-\left|S \cap\left(X_{1} \cup Y_{1}\right)\right|-\mid S \cap\left(X_{2} \cup\right.$ $\left.Y_{2}\right)|\geqslant| X|-| X_{1}|-| X_{2}|=| X_{3} \mid$. It follows that $\left|S \cap X_{3}\right|=\left|X_{3}\right|$. Then $X_{3} \subseteq S$. Hence, $X_{3} \subseteq \bigcap_{S \in \mathcal{F}} S$ by the arbitrary choice of $S$.

Next, suppose there exists a vertex $u \in X_{3}$ with $d_{G}(u)=d>0$. Let $N_{G}(u):=$ $\left\{v_{1}, \ldots, v_{d}\right\}$. Then $\left\{v_{1}, \ldots, v_{d}\right\} \subseteq Y_{2}$. Let $u_{1}, \ldots, u_{d} \in X_{2}$ be such that $u_{i} v_{i} \in E(M)$ for all $i \in[d]$. For each $i \in[d]$, let $M^{i}:=\left(M \backslash u_{i} v_{i}\right) \cup\left\{u v_{i}\right\}, X_{2}^{i}:=V\left(M^{i}\right) \cap X$ and $X_{3}^{i}:=X \backslash\left(X_{1} \cup X_{2}^{i}\right)$. Then $u_{i} \in X_{3}^{i}$ and $M^{i}$ is a perfect matching of $G\left[X_{2}^{i}, Y_{2}\right]$. By the arbitrary choice of $M, u_{i} \in \bigcap_{S \in \mathcal{F}} S$. Therefore, $\left|\bigcap_{S \in \mathcal{F}} S\right| \geqslant\left|\left\{u_{1}, \ldots, u_{d}\right\} \cup X_{3}\right| \geqslant$ $d+(2 \alpha(G)-|G|) \geqslant \delta(G)+2 \alpha(G)-|G| \geqslant \delta(G)+1$, as desired.

Finally, if $\bigcap_{S \in \mathcal{F}} S=\{u\}$, then $1=\left|\bigcap_{S \in \mathcal{F}} S\right| \geqslant d+2 \alpha(G)-|G|$. It follows that $d=0$ and $\alpha(G)=(|G|+1) / 2$, because $2 \alpha(G)-|G|>0$.

This completes the proof of Lemma 12. 
We are now ready to prove Theorem 8 .

Proof of Theorem 8: Let $G$ be a $\left(K_{t}, \mathcal{T}_{k}\right)$-co-critical graph on $n$ vertices, where $t \geqslant 4$ and $k \geqslant \max \{6, t\}$. Then $n \geqslant(t-1)(k-1)+1$ and $G$ admits a critical coloring. Among all critical colorings of $G$, let $\tau: E(G) \rightarrow$ \{red, blue $\}$ be a critical coloring of $G$ with $\left|E_{r}\right|$ maximum. By the choice of $\tau, G_{r}$ is $K_{t}$-saturated and $G_{b}$ is $\mathcal{T}_{k}$-free. By Theorem 7 (a), $\delta\left(G_{r}\right) \geqslant 2 t-4$. Let $D_{1}, \cdots, D_{p}$ be all components of $G_{b}$. By Lemma 10(a), $\left|D_{i}\right| \leqslant k-1$ for all $i \in[p]$. Then $(t-1)(k-1)+1 \leqslant n \leqslant p(k-1)$. This implies that $p \geqslant t$. Let $H:=G \backslash\left(\bigcup_{i \in[p]} E\left(G\left[V\left(D_{i}\right)\right]\right)\right)$. Then $H$ is a spanning subgraph of $G_{r}$. Clearly, $H$ is $K_{t}$-free.

Assume first that $\delta(H) \geqslant 2 t-4$. By Theorem 7(h) applied to $H$ and $q=2 t-4$, there exists a constant $c(2 t-4, k)$ such that $e(H) \geqslant(2 t-4) n-c(2 t-4, k)$. This, together with Theorem $7(\mathrm{f})$, yields that

$$
\begin{aligned}
e(G) & =e(H)+\sum_{i=1}^{p} e\left(G\left[V\left(D_{i}\right)\right]\right) \\
& \geqslant(2 t-4) n-c(2 t-4, k)+\left(\frac{1}{2}\left\lceil\frac{k}{2}\right\rceil-\frac{1}{2}\right)(n-(t-1)(\lceil k / 2\rceil-1)) \\
& =\left(\frac{4 t-9}{2}+\frac{1}{2}\left\lceil\frac{k}{2}\right\rceil\right) n-c(2 t-4, k)-\frac{1}{2}(t-1)(\lceil k / 2\rceil-1)^{2} \\
& =\left(\frac{4 t-9}{2}+\frac{1}{2}\left\lceil\frac{k}{2}\right\rceil\right) n-c_{1}(t, k),
\end{aligned}
$$

as desired, where $c_{1}(t, k)=c(2 t-4, k)+\frac{1}{2}(t-1)(\lceil k / 2\rceil-1)^{2}$.

Assume next that $\delta(H) \leqslant 2 t-5$. Note that $k \geqslant \max \{6, t\} \geqslant t$ for all $t \geqslant 4$. Let $u \in$ $V(H)$ with $d_{H}(u)=\delta(H)$. We may assume that $u \in V\left(D_{p}\right)$. Let $N_{H}(u)=\left\{u_{1}, \ldots, u_{\delta(H)}\right\}$. By Theorem 7(b) applied to $u$ and any vertex in $V(H) \backslash\left(V\left(D_{p}\right) \cup N_{H}(u)\right)$, we see that $H\left[N_{H}(u)\right]$ must contain $K_{t-2}$ as a subgraph. We may assume that $H\left[\left\{u_{1}, \ldots, u_{t-2}\right\}\right]=$ $K_{t-2}$. Then we may further assume that $u_{i} \in V\left(D_{p-i}\right)$ for all $i \in[t-2]$. Note that $H\left[N_{H}(u)\right]$ is $K_{t-1}$-free and $\omega\left(H\left[N_{H}(u)\right]\right)=t-2>\left|N_{H}(u)\right| / 2$. Let $\mathcal{F}$ be the family of all $K_{t-2}$ subgraphs of $H\left[N_{H}(u)\right]$. By Theorem $7(\mathrm{~d}),\left|\bigcap_{A \in \mathcal{F}} A\right| \leqslant 1$. By Lemma 12 applied to the complement of $H\left[N_{H}(u)\right]$, we have $\left|\bigcap_{A \in \mathcal{F}} A\right|=1$. We may assume that $\bigcap_{A \in \mathcal{F}} A=\left\{u_{1}\right\}$. By Lemma 12 again, $\left|N_{H}(u)\right|=2 t-5, u_{1}$ is complete to $N_{H}(u) \backslash u_{1}$ in $H$ and $u_{1}$ is contained in all $K_{t-2}$ subgraphs of $H\left[N_{H}(u)\right]$. Then $H\left[N_{H}(u) \backslash u_{1}\right]$ is $K_{t-2}$-free. By Theorem 7(b) applied to $u$ and any vertex in $V(H) \backslash\left(V\left(D_{p}\right) \cup N_{H}(u)\right)$, $V(H) \backslash\left(V\left(D_{p}\right) \cup N_{H}(u)\right)$ must be complete to $u_{1}$ in $H$. Thus $\left\{u_{1}\right\}=V\left(D_{p-1}\right)$. By Theorem $7(\mathrm{~h})$ applied to $H$ and $q=2 t-5$, there exists a constant $c(2 t-5, k)$ such that $e(H) \geqslant(2 t-5) n-c(2 t-5, k)$. Recall that $p \geqslant t$. If $p=t$, then $n=(t-1)(k-1)+1$ 
and $\left|V\left(D_{i}\right)\right|=k-1$ for $i \in[p]$ with $i \neq p-1$. In this case,

$$
\begin{aligned}
e(G) & =e(H)+\sum_{i=1}^{p} e\left(G\left[V\left(D_{i}\right)\right]\right) \\
& \geqslant((2 t-5) n-c(2 t-5, k))+(p-1)(k-1)(k-2) / 2 \\
& =((2 t-5) n-c(2 t-5, k))+(n-1)(k-2) / 2 \\
& =(2 t-6+k / 2) n-c(2 t-5, k)-(k-2) / 2 \\
& \geqslant\left(\frac{4 t-9}{2}+\frac{1}{2}\left\lceil\frac{k}{2}\right\rceil\right) n-c_{2}(t, k)
\end{aligned}
$$

for all $k \geqslant 6$, as desired, where $c_{2}(t, k)=c(2 t-5, k)+(k-2) / 2$.

Next assume $p \geqslant t+1$. Since $k \geqslant t,\left|N_{H}(u)\right| \leqslant 2 t-5$, and $G_{r}$ is $K_{t}$-free, by Lemma 10(b), there are at most $t-1$ many $D_{i}$ 's satisfying $u \notin V\left(D_{i}\right)$ and $D_{i} \backslash N_{H}(u)=\varnothing$. We may assume that for all $i \in[p-t], D_{1}, \ldots, D_{p-t}$ are such that $u \notin V\left(D_{i}\right)$ and $D_{i} \backslash N_{H}(u) \neq \varnothing$. By Theorem $7(\mathrm{c}),\left|D_{i}\right|=k-1$ for all $i \in[p-t]$. Thus

$$
\sum_{i=1}^{p} e\left(G\left[V\left(D_{i}\right)\right]\right) \geqslant(p-t)(k-1)(k-2) / 2 .
$$

Note that $n \leqslant(p-1)(k-1)+1$ because $\left\{u_{1}\right\}=V\left(D_{p-1}\right)$ and $\left|D_{i}\right| \leqslant k-1$ for all $i \in[p]$ with $i \neq p-1$. Therefore,

$$
\begin{aligned}
e(G) & =e(H)+\sum_{i=1}^{p} e\left(G\left[V\left(D_{i}\right)\right]\right) \\
& \geqslant((2 t-5) n-c(2 t-5, k))+(p-t)(k-1)(k-2) / 2 \\
& \geqslant((2 t-5) n-c(2 t-5, k))+\frac{1}{2}\left(\frac{n-1}{k-1}-t+1\right)(k-1)(k-2) \\
& =(2 t-6+k / 2) n-c(2 t-5, k)-(k-2)(t k-t-k+2) / 2 \\
& \geqslant\left(\frac{4 t-9}{2}+\frac{1}{2}\left\lceil\frac{k}{2}\right\rceil\right) n-c(2 t-5, k)-\left[(t-1) k^{2}-(3 t-4) k+2 t-4\right] / 2 \\
& =\left(\frac{4 t-9}{2}+\frac{1}{2}\left\lceil\frac{k}{2}\right\rceil\right) n-c_{3}(t, k)
\end{aligned}
$$

for all $k \geqslant 6$, as desired, where $c_{3}(t, k)=c(2 t-5, k)+\left[(t-1) k^{2}-(3 t-4) k+2 t-4\right] / 2$.

Let $\ell(t, k):=\max \left\{c_{1}(t, k), c_{2}(t, k), c_{3}(t, k)\right\}$. Then

$$
e(G) \geqslant\left(\frac{4 t-9}{2}+\frac{1}{2}\left\lceil\frac{k}{2}\right\rceil\right) n-\ell(t, k)
$$

as desired. This completes the proof of Theorem 8 . 


\section{Proof of Theorem 9}

Let $t \in\{4,5\}, k \geqslant 3$ and $n \geqslant(2 t-3)(k-1)+\lceil k / 2\rceil\lceil k / 2\rceil-1$. We will construct a $\left(K_{t}, \mathcal{T}_{k}\right)$-co-critical graph on $n$ vertices which yields the desired upper bound in Theorem 9 .

Let $r, s$ be the remainder and quotient of $n-(2 t-3)(k-1)$ when divided by $\lceil k / 2\rceil$, and let $A:=K_{k-1}$. For each $i \in[t-2]$, let $B_{i}:=K_{k-2}$ and $C_{i}:=K_{k-2}$. Let $H_{1}$ be obtained from disjoint copies of $A, B_{1}, \ldots, B_{t-2}, C_{1}, \ldots, C_{t-2}$ by joining every vertex in $B_{i}$ to all vertices in $A \cup C_{i} \cup B_{j}$ for each $i \in[t-2]$ and all $j \in[t-2]$ with $j \neq i$. Let $H_{2}:=(s-r) K_{\lceil k / 2\rceil} \cup r K_{\lceil k / 2\rceil+1}$ when $k \geqslant 4$, and $H_{2}:=s K_{2} \cup r K_{1}$ when $k=3$. Finally, let $G$ be the graph obtained from $H:=H_{1} \cup H_{2}$ by adding $2 t-4$ new vertices $x_{1}, \ldots, x_{t-2}, y_{1}, \ldots, y_{t-2}$, and then, for each $i \in[t-2]$, joining: $x_{i}$ to every vertex in $V(H)$ and all $x_{j}$; and $y_{i}$ to every vertex in $V(H) \backslash V(A)$ and all $x_{j}$, where $j \in[t-2]$ with $j \neq i$. The construction of $G$ when $t=4$ and $k \geqslant 4$ is depicted in Figure 1, and the construction of $G$ when $t=5$ and $k \geqslant 4$ is depicted in Figure 2 .

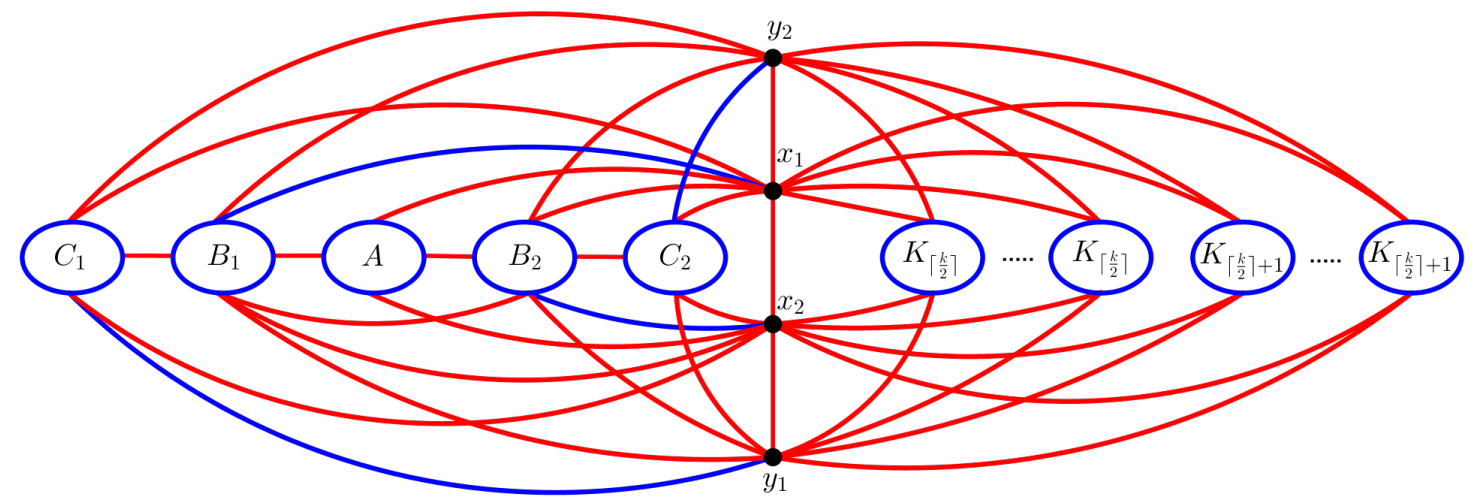

Figure 1: $\mathrm{A}\left(K_{4}, \mathcal{T}_{k}\right)$-co-critical graph for all $k \geqslant 4$.

Let $\sigma: E(G) \rightarrow$ red, blue $\}$ be defined as follows: all edges in $A, B_{1}, \ldots, B_{t-2}$, $C_{1}, \ldots, C_{t-2}$ and $H_{2}$ are colored blue; for every $i \in[t-2]$, all edges between $x_{i}$ and $B_{i}$ are colored blue and all edges between $y_{i}$ and $C_{i}$ are colored blue; the remaining edges of $G$ are all colored red. Note that the $\{$ red, blue $\}$-coloring of $G$ depicted in Figure 1 (resp. Figure 2) is $\sigma$ when $t=4$ (resp. $t=5$ ) and $k \geqslant 4$. It is simple to check that $\sigma$ is a critical coloring of $G$. We next show that $\sigma$ is the unique critical coloring of $G$ up to symmetry.

Let $X:=\left\{x_{1}, \ldots, x_{t-2}\right\}$ and $Y:=\left\{y_{1}, \ldots, y_{t-2}\right\}$. Let $\tau: E(G) \rightarrow\{$ red, blue $\}$ be an arbitrary critical coloring of $G$. It suffices to show that $\tau=\sigma$ up to symmetry. Let $G_{r}^{\tau}$ and $G_{b}^{\tau}$ be $G_{r}$ and $G_{b}$ under the coloring $\tau$, respectively. Note that $G\left[V(A) \cup V\left(B_{1}\right) \cup\right.$ $\left.\cdots \cup V\left(B_{t-2}\right) \cup X\right]=K_{(t-1)(k-1)}$. By Lemma 10 (a) and the fact that $G_{r}^{\tau}$ is $K_{t}$-free, 


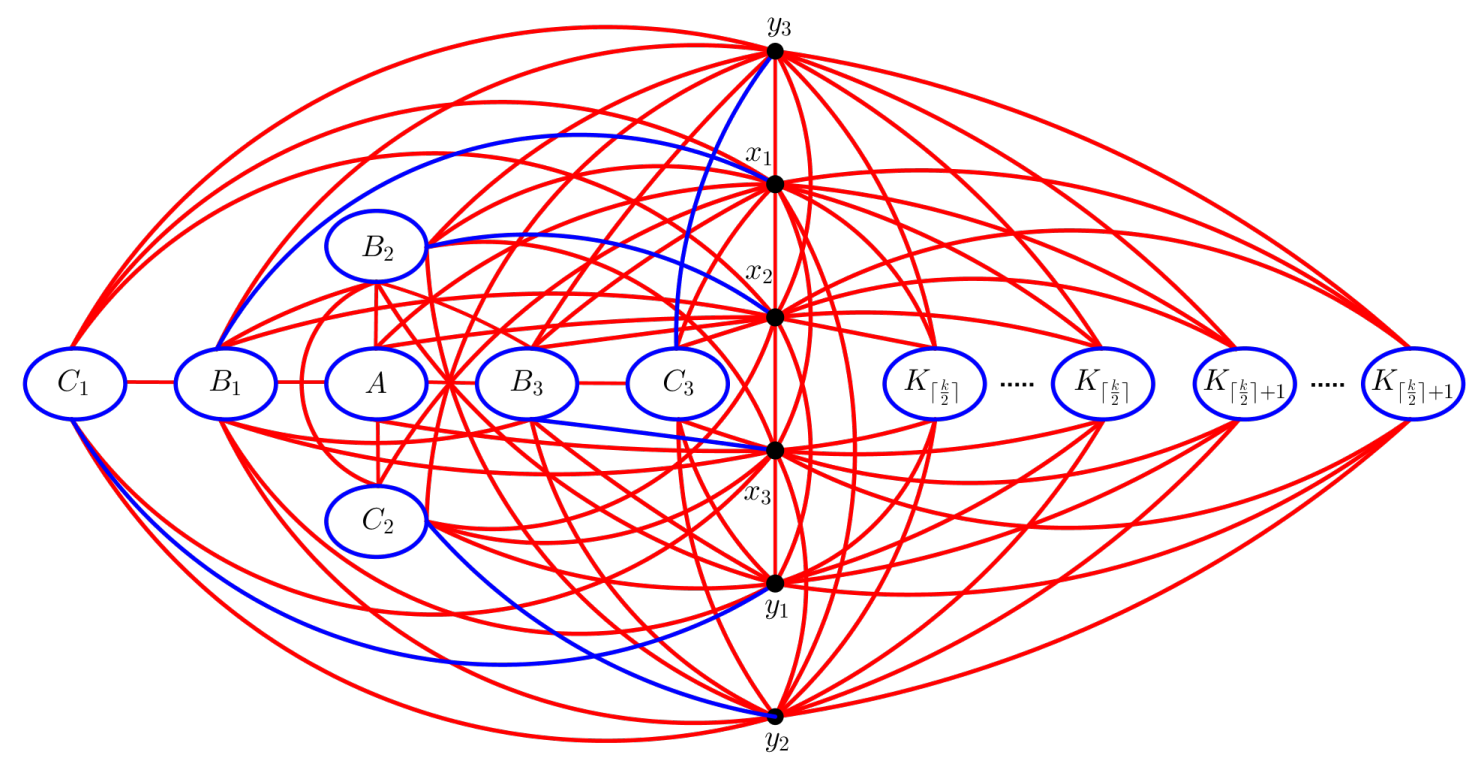

Figure 2: $\mathrm{A}\left(K_{5}, \mathcal{T}_{k}\right)$-co-critical graph for all $k \geqslant 4$.

$G_{b}^{\tau}\left[V(A) \cup V\left(B_{1}\right) \cup \cdots \cup V\left(B_{t-2}\right) \cup X\right]$ has exactly $t-1$ components, say $D_{1}, \ldots, D_{t-1}$, such that $V\left(D_{i}\right)$ is complete to $V\left(D_{j}\right)$ in $G_{r}^{\tau}$ for all $i, j \in[t-1]$ with $i \neq j$. Then each $D_{i}$ is isomorphic to $K_{k-1}$ in $G_{b}^{\tau}$ for all $i \in[t-1]$. Since every vertex in $V(A) \cup V\left(B_{1}\right) \cup$ $\cdots \cup V\left(B_{t-2}\right) \cup X$ belongs to a blue $K_{k-1}$ in $G_{b}^{\tau}$, it follows that: for each $i \in[t-2], y_{i}$ is complete to $V\left(B_{1}\right) \cup \cdots \cup V\left(B_{t-2}\right) \cup\left(X \backslash x_{i}\right)$ in $G_{r}^{\tau}$; and $V\left(C_{i}\right)$ is complete to $V\left(B_{i}\right) \cup X$ in $G_{r}^{\tau}$. We next prove three claims.

Claim 1. $A=D_{i}$ for some $i \in[t-1]$.

Proof. Suppose $A \neq D_{i}$ for all $i \in[t-1]$. Then for each $i \in[t-1]$, we see that $\left(V\left(B_{1}\right) \cup \cdots \cup V\left(B_{t-2}\right) \cup X\right) \cap V\left(D_{i}\right) \neq \varnothing$. Let $d_{i} \in\left(V\left(B_{1}\right) \cup \cdots \cup V\left(B_{t-2}\right) \cup X\right) \cap V\left(D_{i}\right)$ for each $i \in[t-1]$. Then $d_{1}, \ldots, d_{t-1}$ are pairwise distinct and $G_{r}^{\tau}\left[\left\{d_{1}, \ldots, d_{t-1}\right\}\right]=K_{t-1}$. Note that either $X \subseteq\left\{d_{1}, \ldots, d_{t-1}\right\}$ or $x_{i} \notin\left\{d_{1}, \ldots, d_{t-1}\right\}$ for some $i \in[t-2]$. It follows that in the former case, $G_{r}^{\tau}\left[\left\{d_{1}, \ldots, d_{t-1}, u\right\}\right]=K_{t}$ for any $u \in C_{1}$, and in the latter case, $G_{r}^{\tau}\left[\left\{d_{1}, \ldots, d_{t-1}, y_{i}\right\}\right]=K_{t}$, because $y_{i}$ is complete to $V\left(B_{1}\right) \cup \cdots \cup V\left(B_{t-2}\right) \cup\left(X \backslash x_{i}\right)$ in $G_{r}^{\tau}$. In both cases we obtain a contradiction because $G_{t}^{\tau}$ is $K_{t}$-free.

By Claim 1, we may assume that $A=D_{t-1}$. Then $V(A)$ is complete to $V\left(B_{1}\right) \cup \cdots \cup$ $V\left(B_{t-2}\right) \cup X$ in $G_{r}^{\tau}$. For each $i \in[t-2]$, since $G_{b}^{\tau}$ is $\mathcal{T}_{k}$-free, there must exist a vertex $c_{i} \in V\left(C_{i}\right)$ such that $c_{i}$ is adjacent to at most one vertex of $Y$ in $G_{b}^{\tau}$. Then $c_{i}$ is adjacent to at least $t-3$ vertices of $Y$ in $G_{r}^{\tau}$. We next show that

Claim 2. For each $i \in[t-2],\left|X \cap V\left(D_{i}\right)\right|=1$. 
Proof. Suppose $\left|X \cap V\left(D_{i}\right)\right| \neq 1$ for some $i \in[t-2]$. Since $|X|=t-2$, we may assume that $\left|X \cap V\left(D_{1}\right)\right| \geqslant 2$ and $X \cap V\left(D_{t-2}\right)=\varnothing$. We may further assume that $x_{1}, x_{2} \in V\left(D_{1}\right)$. Then $x_{1} x_{2} \in E_{b}$. Since $X \cap V\left(D_{t-2}\right)=\varnothing$ and for all $i \in[t-2]$, $\left|V\left(B_{i}\right)\right|=k-2<k-1=\left|V\left(D_{t-2}\right)\right|$, we may assume that $V\left(B_{i}\right) \cap V\left(D_{t-2}\right) \neq \varnothing$ for $i \in[2]$. Let $b_{1} \in V\left(B_{1}\right) \cap V\left(D_{t-2}\right)$. We see that $c_{1} y_{i} \in E_{r}$ for some $i \in$ [2], because $c_{1}$ is adjacent to at least $t-3$ vertices of $Y$ in $G_{r}^{\tau}$. If $t=4$, then $G_{r}^{\tau}\left[\left\{b_{1}, c_{1}, y_{i}, x_{3-i}\right\}\right]=K_{4}$, a contradiction. Thus $t=5$. Suppose $V\left(B_{1}\right) \cap V\left(D_{2}\right) \neq \varnothing$. Let $b_{2} \in V\left(B_{1}\right) \cap V\left(D_{2}\right)$. Then $G_{r}^{\tau}\left[\left\{b_{1}, b_{2}, c_{1}, y_{i}, x_{3-i}\right\}\right]=K_{5}$, a contradiction. Thus $V\left(B_{1}\right) \cap V\left(D_{2}\right)=\varnothing$. By symmetry, $V\left(B_{2}\right) \cap V\left(D_{2}\right)=\varnothing$. Then $V\left(D_{2}\right)=V\left(B_{3}\right) \cup\left\{x_{3}\right\}$. But then $G_{r}^{\tau}\left[\left\{b_{1}, c_{1}, y_{i}, x_{3-i}, x_{3}\right\}\right]=K_{5}$, a contradiction.

Claim 3. For each $i \in[t-2], V\left(B_{i}\right) \subseteq V\left(D_{j}\right)$ for some $j \in[t-2]$.

Proof. Suppose there exists an $i \in[t-2]$ such that $V\left(B_{i}\right) \nsubseteq V\left(D_{j}\right)$ for every $j \in$ $[t-2]$. We may assume $i=1$. Since $V\left(B_{1}\right) \subseteq V\left(D_{1}\right) \cup \cdots \cup V\left(D_{t-2}\right)$, we see that $k-2=\left|B_{1}\right| \geqslant 2$. Thus $k \geqslant 4$. We claim that $V\left(B_{1}\right) \cap V\left(D_{j}\right)=\varnothing$ for some $j \in$ $[t-2]$. Suppose $V\left(B_{1}\right) \cap V\left(D_{j}\right) \neq \varnothing$ for all $j \in[t-2]$. Let $d_{j} \in V\left(B_{1}\right) \cap V\left(D_{j}\right)$ for all $j \in[t-2]$. But then $G_{r}^{\tau}\left[\left\{d_{1}, \ldots, d_{t-2}, c_{1}, y_{\ell}\right\}\right]=K_{t}$, where $c_{1} y_{\ell} \in E_{r}$ for some $\ell \in[t-2]$, a contradiction. Thus $V\left(B_{1}\right) \cap V\left(D_{j}\right)=\varnothing$ for some $j \in[t-2]$, as claimed. We may assume that $V\left(B_{1}\right) \cap V\left(D_{t-2}\right)=\varnothing$. Since $V\left(B_{1}\right) \nsubseteq V\left(D_{j}\right)$ for every $j \in$ $[t-2]$, it follows that $t=5, V\left(B_{1}\right) \subseteq V\left(D_{1}\right) \cup V\left(D_{2}\right)$, and $V\left(B_{1}\right) \cap V\left(D_{1}\right) \neq \varnothing$ and $V\left(B_{1}\right) \cap V\left(D_{2}\right) \neq \varnothing$. Let $d_{1} \in V\left(B_{1}\right) \cap V\left(D_{1}\right)$ and $d_{2} \in V\left(B_{1}\right) \cap V\left(D_{2}\right)$. By Claim 2, let $x_{i} \in X \cap V\left(D_{3}\right)$. Then $G_{r}^{\tau}\left[\left\{d_{1}, d_{2}, x_{i}, c_{1}, y_{j}\right\}\right]=K_{5}$, where $c_{1} y_{j} \in E_{r}$ for some $j \in[3]$ with $j \neq i$, a contradiction.

By Claim 2 and Claim 3, $V\left(B_{i}\right) \cup V\left(B_{j}\right) \nsubseteq D_{\ell}$ for any $i \neq j \in[t-2]$ and all $\ell \in[t-2]$. By symmetry, we may assume that $V\left(B_{i}\right) \subseteq V\left(D_{i}\right)$ for all $i \in[t-2]$. Then $V\left(B_{i}\right) \cup\left\{x_{j}\right\}=V\left(D_{i}\right)$ for some $j \in[t-2]$ since $\left|V\left(D_{i}\right)\right|=\left|V\left(B_{i}\right)\right|+1$ and $V\left(B_{1}\right) \cup \cdots \cup V\left(B_{t-2}\right) \cup X=V\left(D_{1}\right) \cup \cdots \cup V\left(D_{t-2}\right)$. By symmetry, we may assume that $V\left(B_{i}\right) \cup\left\{x_{i}\right\}=V\left(D_{i}\right)$ for all $i \in[t-2]$. It follows that for all $i, j \in[t-2]$ with $i \neq j$, $B_{i}$ is complete to $B_{j}$ in $G_{r}^{\tau}, x_{i}$ is complete to $X \backslash x_{i}$ and $B_{j}$ in $G_{r}^{\tau}, y_{i}$ is complete to $C_{i}$ in $G_{b}^{\tau}, y_{i}$ is complete to $C_{j} \cup\left(X \backslash x_{i}\right)$ in $G_{r}^{\tau}, x_{i}$ is complete to $B_{i}$ in $G_{b}^{\tau},\left\{x_{i}, y_{i}\right\}$ is complete to $H_{2}$ in $G_{r}^{\tau}$, all edges in $A, B_{1}, \ldots, B_{t-2}, C_{1}, \ldots, C_{t-2}$ and $H_{2}$ are colored blue under $\tau$. This proves that $\tau=\sigma$ and thus $\sigma$ is the unique critical coloring of $G$ up to symmetry. It can be easily checked that adding any edge $e \in E(\bar{G})$ to $G$ creates a red $K_{t}$ if $e$ is colored red, and a blue $T_{k}$ if $e$ is colored blue. Hence, $G$ is $\left(K_{t}, \mathcal{T}_{k}\right)$-co-critical. Note that

$$
\begin{aligned}
e_{G}(X \cup Y, V(G) \backslash(X \cup Y)) & =(t-2)(n-(2 t-4))+(t-2)(n-(2 t-4+k-1)) \\
& =(t-2)(2 n-4 t-k+9) ;
\end{aligned}
$$


$e_{G}(X \cup Y)=\left(\begin{array}{c}t-2 \\ 2\end{array}\right)+(t-2)(t-3) ; e_{G}\left(V\left(B_{1}\right) \cup \cdots \cup V\left(B_{t-2}\right), V\left(C_{1}\right) \cup \cdots \cup V\left(C_{t-2}\right)\right)=$ $(t-2)(k-2)^{2} ; e_{G}\left(V\left(C_{1}\right) \cup \cdots \cup V\left(C_{t-2}\right)\right)=(t-2)\left(\begin{array}{c}k-2 \\ 2\end{array}\right) ; e_{G}\left(V(A) \cup V\left(B_{1}\right) \cup \cdots \cup V\left(B_{t-2}\right)\right)=$ $\left(\begin{array}{c}(t-2)(k-2)+k-1 \\ 2\end{array}\right)$. Using the facts that $s\lceil k / 2\rceil+r=n-(2 t-3)(k-1)$ and $r \leqslant\lceil k / 2\rceil-1$, we see that when $k \geqslant 4$,

$$
\begin{aligned}
e(G)= & (t-2)(2 n-4 t-k+9)+\left(\begin{array}{c}
t-2 \\
2
\end{array}\right)+(t-2)(t-3)+(t-2)(k-2)^{2}+ \\
& (t-2)\left(\begin{array}{c}
k-2 \\
2
\end{array}\right)+\left(\begin{array}{c}
(t-2)(k-2)+k-1 \\
2
\end{array}\right)+(s-r)\left(\begin{array}{c}
\lceil k / 2\rceil \\
2
\end{array}\right)+r\left(\begin{array}{c}
\lceil k / 2\rceil+1) \\
2
\end{array}\right) \\
= & (2 t-4) n-(t-2) k-\frac{1}{2}(t-2)(5 t-9) \\
& +(k-2)((t-2)(k-2)+(t-2)(k-3) / 2+(t-1)(t k-k-2 t+3) / 2) \\
& +\frac{s-r}{2}\left\lceil\frac{k}{2}\right\rceil\left(\left\lceil\frac{k}{2}\right\rceil-1\right)+\frac{r}{2}\left\lceil\frac{k}{2}\right\rceil\left(\left\lceil\frac{k}{2}\right\rceil+1\right) \\
= & (2 t-4) n-(t-2)\left(k-\frac{1}{2}(5 t-9)\right)+\frac{1}{2}(k-2)\left(\left(t^{2}+t-5\right) k-2 t^{2}-2 t+11\right) \\
& +\frac{1}{2}\left(\left\lceil\frac{k}{2}\right\rceil-1\right)\left(s\left\lceil\frac{k}{2}\right\rceil+r\right)+\frac{r}{2}\left(\left\lceil\frac{k}{2}\right\rceil+1\right) \\
\leqslant & (2 t-4) n+\frac{1}{2}\left(\left(t^{2}+t-5\right) k^{2}-\left(4 t^{2}+6 t-25\right) k-t^{2}+23 t-40\right) \\
& +\frac{1}{2}\left(\left\lceil\frac{k}{2}\right\rceil-1\right)(n-(2 t-3)(k-1))+\frac{1}{2}\left(\left\lceil\frac{k}{2}\right\rceil-1\right)\left(\left\lceil\frac{k}{2}\right\rceil+1\right) \\
= & \left(\frac{4 t-9}{2}+\frac{1}{2}\left\lceil\frac{k}{2}\right\rceil\right) n+\frac{1}{2}\left(t^{2}+t-5\right) k^{2}-\left(2 t^{2}+2 t-11\right) k \\
& -\frac{(t-2)(t-19)}{2}-\frac{1}{2}\left\lceil\frac{k}{2}\right\rceil\left((2 t-3)(k-1)-\left\lceil\frac{k}{2}\right\rceil\right) \\
= & \left(\frac{4 t-9}{2}+\frac{1}{2}\left\lceil\frac{k}{2}\right\rceil\right) n+C(t, k),
\end{aligned}
$$

where $C(t, k)=\frac{1}{2}\left(t^{2}+t-5\right) k^{2}-\left(2 t^{2}+2 t-11\right) k-\frac{(t-2)(t-19)}{2}-\frac{1}{2}\left\lceil\frac{k}{2}\right\rceil\left((2 t-3)(k-1)-\left\lceil\frac{k}{2}\right\rceil\right)$.

When $k=3$, we have $2 s \leqslant n-2(2 t-3)$ and

$$
\begin{aligned}
e(G) & =(t-2)(2 n-4 t-3+9)+\left(\begin{array}{c}
t-2 \\
2
\end{array}\right)+(t-2)(t-3)+(t-2)+\left(\begin{array}{c}
(t-2)+2 \\
2
\end{array}\right)+s \\
& \leqslant(2 t-4) n-(t-2)(3 t-4)+\left(\begin{array}{c}
t-2 \\
2
\end{array}\right)+\left(\begin{array}{l}
t \\
2
\end{array}\right)+\frac{n-2(2 t-3)}{2} \\
& =(2 t-7 / 2) n+\left(\begin{array}{c}
t-2 \\
2
\end{array}\right)+\left(\begin{array}{l}
t \\
2
\end{array}\right)-\left(3 t^{2}-8 t+5\right) \\
& =(2 t-7 / 2) n+C(t, 3),
\end{aligned}
$$

where $C(t, 3)=\left(\begin{array}{c}t-2 \\ 2\end{array}\right)+\left(\begin{array}{l}t \\ 2\end{array}\right)-\left(3 t^{2}-8 t+5\right)=-2 t^{2}+5 t-2$.

This completes the proof of Theorem 9 . 


\section{Acknowledgements}

The authors would like to thank both referees for their valuable comments. The first author would like to thank Hehui Wu, from Fudan University, China, for helpful discussion on the statement of Lemma 12 and his neat proof of Lemma 12, which is completely different from the one of Hajnal [11].

\section{References}

[1] B. Bollobás, On generalized graphs, Acta Math. Acad. Sci. Hungar. 16 (1965) 447452.

[2] C. Bosse, Z-X. Song and J. Zhang, On the size of $\left(K_{3}, K_{4}\right)$-co-critical graphs, in preparation.

[3] J. Chalupa, P. L. Leath and G. R. Reich, Bootstrap percolation on a bethe latice, J. Physics C: Solid State Phys. 12 (1979) L31-L37.

[4] V. Chvátal, Tree-complete graph Ramsey numbers, J. Graph Theory 1 (1977) 93.

[5] G. Chen, M. Ferrara, R. J. Gould, C. Magnant and J. Schmitt, Saturation numbers for families of Ramsey-minimal graphs, J. Comb. 2 (2011) 435-455.

[6] A. N. Day, Saturated graphs of prescribed minimum degree, Combin., Probab., Comput 26 (2017) 201-207.

[7] P. Erdős, A. Hajnal and J. W. Moon, A problem in graph theory, Amer. Math. Monthly. 71 (1964) 1107-1110.

[8] J. R. Faudree, R. J. Faudree and J. R. Schmitt, A survey of minimum saturated graphs, Electron. J. Combin. 18 (2011) \#DS19.

[9] M. Ferrara, J. Kim and E. Yeager, Ramsey-minimal saturation numbers for matchings, Discrete Math. 322 (2014) 26-30.

[10] A. Galluccio, M. Simonovits and G. Simonyi, On the structure of co-critical graphs, Graph theory, combinatorics, and algorithms, Vol. 1,2 (Kalamazoo, MI, 1992), 10531071, Wiley-Intersci. Publ., Wiley, New York, 1995.

[11] A. Hajnal, A theorem on $k$-saturated graphs, Canad. J. Math. 17 (1965) 720-724.

[12] D. Hanson and B. Toft, Edge-colored saturated graphs, J. Graph Theory 11 (1987) 191-196.

[13] J. Nešetřil, Problem, in Irregularities of Partitions, (eds G. Halász and V. T. Sós), Springer Verlag, Series Algorithms and Combinatorics, vol 8, (1989) P164. (Proc. Coll. held at Fertőd, Hungary 1986).

[14] M. Rolek and Z-X. Song, Saturation numbers for Ramsey-minimal graphs, Discrete Math. 341 (2018) 3310-3320.

[15] T. Szabó, On nearly regular co-critical graphs, Discrete Math. 160 (1996) 279-281. 